Rev Biomed 2001; 12:11-17.

\title{
Tasa de mortalidad verdadera por estrato de edad y causa de muerte en un rebaño de cabras criollas en el trópico subhúmedo de Yucatán, México.
}

\section{Artículo Original}

Juan F. Torres-Acosta ${ }^{1}$, Armando Aguilar-Caballero ${ }^{1}$, José Williams ${ }^{2}$, Antonio Ortega-Pacheco ${ }^{1}$.

${ }^{1}$ Area de Pequeños Rumiantes, ${ }^{2}$ Depto. de Epidemiología, Facultad de Medicina Veterinaria y Zootecnia, Universidad Autónoma de Yucatán, Mérida, Yucatán, México.

\section{RESUMEN.}

Introducción. La evaluación precisa de la mortalidad de los rebaños caprinos durante el período que estos permanecen en cada una de las etapas de su vida puede ser utilizado para desarrollar estrategias de manejo para reducir la pérdida de animales por etapa productiva o por enfermedad específica.

Materiales y métodos. Se determinó la tasa de mortalidad verdadera (TMV) por estrato de edad y por causa de muerte (TMVC) en un rebaño caprino del estado de Yucatán, México. Se analizaron los registros de mortalidad, crecimiento y ventas ocurridos en los años de 1986 a 1993 (201 muertes). Se calcularon la TMV y la tasa de riesgo (TR) para los estratos de predestete, crecimiento y adulta. También se calculó la TMVC y la TR por causa de muerte ocurrida en los diferentes estratos.

Resultados. La mayor TMV se encontró en el estrato de predestete (2.00 muertos/1000 animales día a riesgo, (ADAR)), seguido por las adultas (0.20 muertos/1000 ADAR) y la etapa de crecimiento (0.17 muertos/1000 ADAR). El IC (95\%) mostró que la TMV entre los estratos de adultas y crecimiento no fueron diferentes. Para las causas de muerte por estrato, la de mayor TMVC en predestete fue la hipotermia (1.20 muertos/1000 ADAR) con una TR de 0.068; para crecimiento fue la parasitosis gastrointestinal (0.09 muertos/1000 ADAR) con una TR de 0.049; y para el estrato de adultas el traumatismo (0.09 muertos/1000 ADAR) con una TR de 0.250.

Discusión. El período de mayor riesgo de muerte fue el predestete. La causa de muerte más importante fue la hipotermia la cual podría ser evitada a través de manejo adecuado.

(Rev Biomed 2001; 12:11-17)

Palabras clave: Cabra, tasa de mortalidad

Solicitud de sobretiros: M. en C. Juan F. Torres-Acosta, Area de Pequeños Rumiantes, Fac. de Medicina Veterinaria y Zootecnia, Universidad Autónoma de Yucatán, Km. 15.5 Carr. Mérida-Xmatkuil, Apdo. Postal 4-116 Itzimná, C.P. 97100, Mérida, Yucatán, México. Tel. (9) 942-32-00 Fax: (9) 942-32-05 E-mail: tacosta@tunku.uady.mx

Recibido el 20/Julio/1999. Aceptado para publicación el 4/Septiembre/2000. 
JF Torres-Acosta, A Aguilar-Caballero, J Williams, A Ortega-Pacheco.

verdadera, trópico.

\section{SUMMARY.}

True mortality rate by age strata and death causes in a criollo goat flock in the subhumid tropics of Yucatan, Mexico.

Introduction. Precise evaluation of the mortality of animals during each stage of their lives can be used to develop a management strategy to reduce animal losses either in each productive stages or for specific disease.

Material and methods. The true mortality rate was determined by age class (TMV) and by cause of death (TMVC) from a goat flock in Yucatán, México. Data of mortality, growth, and sales, from 1986 to 1993 (201 deaths) were analyzed. The TMV and the risk rare (RR was calculated from pre-weaning, juvenile and adult classes. TMVC and related RR were also calculated for each age class.

Results. The highest TMV was found in the preweaning class [2.00 deaths/1000 animal days at risk (ADAR)], followed by adults ( 0.20 deaths/ 1000 ADAR) and growing class (0.17 deaths/ 1000 ADAR). The $95 \%$ IC showed that TMV between the adult and growing classes were not different. Hypothermia had the highest TMVC (1.20 deaths/1000 ADAR) in the pre-weaning class with a RR of 0.068 . In the juvenile group, the main cause of death was gastrointestinal parasitsm (0.09 deaths/1000 ADAR) with a TR of 0.49 . In the adult class, traumatism was important (0.09 deaths/1000 ADAR) with a TR of 0.250 .

Discussion. The pre-weaning was the period of the highest risk of death. Hypothermia was the most important cause of death which could be avoided by good management.

(Rev Biomed 2001; 12:11-17)

Key words: Goat, true mortality rate, tropics.

\section{INTRODUCCION.}

La mortalidad presente en los rebaños caprinos en el trópico es resultado de la combinación de los efectos negativos de los factores climáticos, las enfermedades y el deficiente manejo de los animales en sus etapas productivas (predestete, crecimiento y adulta) (1). Cuando las cabras no reciben el manejo adecuado en dichas etapas se vuelven susceptibles a enfermedades que pueden propiciar una disminución en su producción o su muerte; lo que ocasiona una reducción en la eficiencia productiva del rebaño. La identificación de las causas de mortalidad en un rebaño permite dictar medidas correctivas y preventivas que mejoren la eficiencia productiva del mismo (2). En el sureste de México, particularmente en Yucatán, se han realizado algunos estudios sobre las prevalencias de diferentes enfermedades y de causas de mortalidad en caprinos $(3,4)$. Estos trabajos se refieren al impacto de la mortalidad encontrada en los rebaños de acuerdo a las causas o a los estratos afectados por separado. Los resultados de dicha mortalidad se presentan en proporciones tal como ocurre en reportes a nivel nacional e internacional (1,5-7). Sin embargo, los datos obtenidos son poco precisos y no permiten identificar el momento en la vida del animal en que existe mayor probabilidad de fallecer por este problema. Por otra parte la tasa de mortalidad verdadera (TMV), la tasa de riesgo (TR) y el intervalo de confianza (IC) son otras herramientas que pueden utilizarse para el análisis de mortalidad en los rebaños caprinos. La TMV describe la velocidad promedio a la cual el evento de interés (muerte) se produce por unidad de tiempo (8). La TR es un estimador directo de la probabilidad de que un animal sufra el evento de interés durante un período dado (9). El IC estima los límites en que se encuentran las TMV para el estrato en estudio (8). Estos permiten una evaluación precisa de la mortalidad de los animales durante el período que éstos permanecen en cada una de las etapas de su vida. Estos parámetros nos permitirán desarrollar estrategias de manejo para

\section{Revista Biomédica}




\section{Mortalidad de cabras en Yucatán, México.}

reducir la pérdida de animales por etapa productiva o por enfermedad específica (10).

El objetivo del presente trabajo fue medir la TMV y la TR por estrato de edad y por causa de muerte en un rebaño caprino de la zona centro del estado de Yucatán.

\section{MATERIALES Y METODOS.}

El presente trabajo se realizó con los registros de mortalidad de un rebaño de la zona centro del estado de Yucatán, ocurrida durante los años de 1986 a 1993. La unidad está ubicada en la zona centro del estado de Yucatán a $20^{\circ} 58^{\prime}$ latitud norte y $89^{\circ} 37^{\prime}$ longitud oeste. En esta zona se presenta una precipitación pluvial anual promedio de $927.8 \mathrm{~mm}$. Con dos épocas, una de lluvias (mayo-octubre) y otra de secas (noviembre-mayo). La temperatura media anual es de $26^{\circ} \mathrm{C}$ con una humedad relativa promedio de $80 \%$ (11). Esta unidad fue elegida porque contaba con los registros precisos de producción y mortalidad.

La población promedio del rebaño durante el período estudiado fue de 376.5 animales (19861993). Para el estudio, los animales fueron agrupados en tres estratos de edad: a) predestete (nacimiento hasta 60 días de edad, b) crecimiento (61 hasta 580 días de edad) y c) adultos (mayores de 580 días de edad). La información se capturó y se analizó en una base de datos computarizada (12).

Los indicadores obtenidos se calcularon con las siguientes fórmulas:

$$
\begin{aligned}
& T M V=\frac{\text { Total de muertos en el estrato }}{T A R} \times 1000 A D A R_{(8,10)} \\
& \text { TAR }=\text { Tiempo Animal a Riesgo del estrato (8) } \\
& \text { ADAR }=\text { Animales días a riesgo. }
\end{aligned}
$$

La fórmula para el cálculo de la tasa de mortalidad verdadera por causa (TMVC) fue similar a la de TMV, pero en este caso, el numerador fue el número de animales muertos por cada una de las causas en el estrato.
La TR por estrato se calculó de acuerdo a Giesecke (9):

$\mathrm{TR}=1-\mathrm{e}^{-(\mathrm{TMV} * \text { Tiempo) }}$

El IC se calculó de acuerdo a Rothman (8):

EE $\ln$ incidencia $\left.=\sqrt{(} \frac{1}{a}\right)$

$\mathrm{EE}=$ error estándar.

$\ln =$ logaritmo natural.

$\mathrm{a}=$ número de eventos.

El 95\% de IC de la incidencia es:

In de la incidencia \pm 1.96 Error Estándar. El 95\% de IC de la incidencia se obtiene tomando los anti-logaritmos de los limites del ln de la incidencia (8). El IC se calculó solamente en las etapas donde el número de datos fue mayor a 10 eventos.

\section{RESULTADOS.}

Durante el período de estudio se registraron 201 animales muertos. De los cuales $103(51.24 \%)$ fueron del estrato de predestete, $37(18.41 \%)$ de crecimiento y $61(30.35 \%)$ de adultos. La mayor TMV se encontró en el estrato de predestete (1.90 muertos/1000 ADAR) (cuadro1). La TR para el estrato adulto fue la mayor. En cuanto a las causas de muerte en el estrato de predestete, las causas con mayor TMVC fueron la hipotermia (1.20 muertos/1000 ADAR) y la hipoglucemia (0.40 muertos/1000 ADAR), que en conjunto representan el $84 \%$ de la mortalidad encontrada (cuadro 2). En el estrato de crecimiento la parasitosis presentó la mayor TMVC (0.097 muertos/1000 ADAR) seguida por el traumatismo (0.056 muertos/1000 ADAR) (cuadro 2). Para el estrato de adultas la causa con mayor TMVC fue el traumatismo (0.098 muertos/1000 ADAR) seguida por la parasitosis (0.046 muertos/1000 ADAR) (cuadro 2). 
JF Torres-Acosta, A Aguilar-Caballero, J Williams, A Ortega-Pacheco.

Cuadro 1

Tasa de mortalidad verdadera (TMV), tiempo animales a riesgo (TAR) y tasa de riesgo (TR) por estrato de un rebaño caprino en la zona centro del estado de Yucatán, México.

\begin{tabular}{lrcccl}
\hline Estrato & $\mathrm{n}$ & TAR & TMV & IC 95\% & TR \\
\hline Predestete & 103 & 54,237 & 1.90 & $1.60-2.40$ & 0.10 \\
Crecimiento & 37 & 215,758 & 0.17 & $0.12-0.23$ & 0.08 \\
Adulta & 61 & 305,140 & 0.20 & $0.15-0.26$ & 0.44 \\
\hline
\end{tabular}

$\mathrm{n}=$ Número de animales a riesgo

IC $95 \%$ = Intervalo de confianza $95 \%$

\section{DISCUSION.}

Los resultados obtenidos del análisis de la TMV no pueden ser comparados con la literatura debido a que no existen autores que reporten este tipo de datos. Sin embargo, el patrón de conducta de la mortalidad sí es comparable. Al respecto Aguilar (13), Torres y col. (4) y Lodh y col. (18), menciona que la mortalidad de los animales en sus diferentes etapas fisiológicas es similar entre las diferentes especies de interés zootécnico, siendo mayor en el estrato de predestete seguida por la etapa adulta y finalmente por la etapa de crecimiento.

Existen reportes de mortalidad proporcional para cabras de diferentes razas, climas y sistemas de producción con resultados similares al presente estudio $(1,4,5)$. Por ejemplo, MacK (14), reportó una mortalidad para la etapa de predestete de $33 \%$ y de $23 \%$ para crecimiento. Falcon y col. (5), por su parte reportaron mortalidades de $16.7-37.7 \%$ en predestete y de 2.9-13.6\% en cabras en etapa adultas. Opasina (15) por su parte, menciona que la mortalidad de los cabritos puede llegar a ser de hasta un $50 \%$ en el trópico. Este comportamiento podría ser por deficiencias en el manejo (nutricional, instalaciones, reproducción y sanitario) que reciben los animales durante su vida productiva, en otras palabras, el sistema de producción es determinante $(6,7,16,17,19,20)$. Una alta mortalidad en adultos no es tan común como en predestete, pero puede ocurrir por brotes de enfermedades, sobre todo cuando el rebaño presenta una alimentación deficiente o problemas crónicos de salud (21).

La TMV encontrada para el estrato de predestete fue superior al de adultas, sin embargo, la TR fue inferior en la primera. Este comportamiento podría explicarse por la amplitud del período de observación para cada animal del estrato de adultas (2,920 días) en comparación al de predestete (60 días), implicando un mayor tiempo a riesgo para

\section{Cuadro 2}

Tasa de mortalidad verdadera por causa (TMVC), tiempo animales a riesgo (TAR) y tasa de riesgo (TR) en los estratos de predestete, crecimiento y adultas de un rebaño caprino de la zona centro del estado de Yucatán, México.

\begin{tabular}{lcccccc}
\hline ESTRATO & CAUSA & $\mathrm{n}$ & TAR & TMVC & IC 95\% & TR \\
\hline Predestete & Hipotermia & 64 & 54,237 & 1.20 & $0.93-1.50$ & 0.068 \\
& Hipoglucemia & 22 & & 0.40 & $0.29-0.60$ & 0.024 \\
& Traumatismo & 10 & & 0.18 & $0.10-0.34$ & 0.011 \\
\multirow{2}{*}{ Crecimiento } & Parasitosis & 21 & 215,758 & 0.097 & $0.027-0.150$ & 0.049 \\
& Traumatismo & 12 & & 0.056 & $0.032-0.099$ & 0.028 \\
\multirow{2}{*}{ Adulta } & Traumatismo & 30 & \multirow{2}{*}{305,140} & 0.098 & $0.069-0.140$ & 0.250 \\
& Parasitosis & 14 & & 0.046 & $0.027-0.078$ & 0.120 \\
\hline
\end{tabular}

$\mathrm{n}=$ Número de animales a riesgo

IC $95 \%$ = Intervalo de confianza $95 \%$

Revista Biomédica 
Mortalidad de cabras en Yucatán, México.

animales adultos. La TMV para el estrato de crecimiento fue la más baja en este estudio. Debido a que el IC (95\%) obtenido para los estratos de adulta y crecimiento fueron similares, la TMV entre estos podría ser semejante (cuadro 1). La etapa de crecimiento es reconocida como el período de mayor supervivencia en los seres vivos, en esta, los órganos vitales alcanzan su máxima capacidad funcional y permite al animal afrontar con éxito los problemas del medio ambiente físico (climatológicos) o infecciosos, por sus características genéticas o por su fácil adaptación al medio ambiente que lo rodea (22,23); esto podría explicar el comportamiento observado en este estudio. En cuanto a las causas de muerte, para el estrato de predestete las de mayor TMVC fueron la hipotermia (1.20 muertos/1000 ADAR) y la hipoglucemia (0.40 muertos/1000 ADAR), que en conjunto representaron el $84 \%$ de la mortalidad encontrada. Referente a esto Torres y Aguilar (2) y Slee (23), mencionan que los cabritos al nacimiento presentan un pobre desarrollo inmunológico y esto los puede hacer susceptibles a morir cuando las condiciones climáticas, de alimentación y manejo son adversas. Esto explica en parte la mortalidad de los cabritos por hipotermia en los primeros días de vida (primeras $48 \mathrm{~h}$ de vida) e hipoglucemia ( 2 a 7 días de vida). En el estrato de crecimiento el parasitismo gastrointestinal (PGI) presentó la mayor TMVC (0.097 muertos/1000 ADAR). El PGI es reconocido como uno de los principales factores que influyen negativamente en el comportamiento productivo de los pequeños rumiantes $(7,20,24)$. Sus efectos pueden ser una reducción en la ganancia de peso, disminución en la producción de leche (25) y la muerte (26). A su vez, Torres y col. $(4,26)$, reportan que en el estado de Yucatán los PGI de mayor importancia son: Eimerias (coccidias) y nemátodos como el Haemonchus contortus. No obstante que en el rebaño se llevaba a cabo el control químico de los parásitos su presencia fue persistente. Esta situación pudo estar influenciada por el medio ambiente, lugar de pastoreo, humedad, carga animal y/o la presencia de nemátodos resistentes a los antihelmínticos (27), por el mal uso de los desparasitantes (28). Para el estrato de adultos la causa con mayor TMVC fue el traumatismo (0.098 muertos/1000 ADAR) seguida por la parasitosis (0.046 muertos/1000 ADAR). Los traumatismos en las cabras adultas es resultado de deficiencias en el manejo de espacio vital en los corrales de estancia, de insuficientes comederos y bebederos, así como por la introducción de nuevos animales al rebaño $(2,4)$. En este estudio estas condiciones se presentaron que junto con los traumatismos sufridos por las cabras atropelladas por vehículos y caídas en zanjas; explican la alta TMVC encontrada para esta causa. Las cabras son una especie que no presenta respuestas inmunitarias adecuadas (premunidad) a los nemátodos gastrointestinales (29). Esto las hace susceptibles en su etapa adulta a los parásitos hematófagos como $H$. contortus (26) cuyos efectos patofisiológicos como la anemia, pueden influir en su producción de leche o en su sobrevivencia (30). Se concluye que el período de mayor riesgo de muerte fue el predestete. La causa de muerte más importante fue la hipotermia. La supervisión de los partos y del recién nacido de manera que se asegure el consumo de calostro y la estancia en parideros con camas de materiales termoaislantes como paja o viruta ayudarían a prevenir la hipotérmia y la hipoglucemia lo cual mejoraría la sobrevivencia de cabritos en el rebaño.

\section{REFERENCIAS.}

1.- Maiga S. Small ruminant morbidity and mortality in the delta of Niger, Mali. Small Rumin Res 1992; 9:1818.

2.- Torres AJF, Aguilar CAJ. Principios de zootécnia de caprinos. Dpto. de Pequeños Rumiantes. Fac. de Med. Vet. y Zoot. Mérida: Universidad Autónoma de Yucatán; 1995. p. $10-45$. 


\section{JF Torres-Acosta, A Aguilar-Caballero, J Williams, A Ortega-Pacheco.}

3.- Bazán J. Situación actual de la caprinocultura en la ciudad de Mérida Yucatán. Tesis. Lic. en MVZ. Fac. de Med. Vet. y Zoot. Universidad Autónoma de Yucatán. Mérida, Yucatán, México. 1985.

4.- Torres AJF, Aguilar CAJ, Ayil OJ. Principales causas de mortalidad de cabras Criollas en trópico subhúmedo del estado de Yucatán. Memorias de la XI reunión nacional sobre caprinocultura. UACH-AMPCA. Edo. Méx., México; 1996. p. 155-8.

5.- Falcon R., Salinas G, Avila R, Flores R. Problemática sanitaria de los caprinos en Zacatecas. Memorias de la VII Reunión Nacional sobre Caprinocultura. FMVZUANL, Monterrey, NL, Méx; 1991. p. 132-4.

6.- Medeiros LFD, Souza JCD, Coutinho LS, Liseu LC, Batista LB. A comparative study of growth in AngloNubian, SRD (nondescript) and Anglo-Nubian X SRD kids. Arquivos-da-Universidade-Federal-Rural-do-Riode-Janeiro 1992; 15:7-19.

7.- Ikwuegbu OA, Tarawali G, Rege J.EO. Effects of fodder banks on growth and survival of West African Dwarf goats under village conditions in subhumid Nigeria. Smal. Rumin Res 1995; 17:101-9.

8.- Rothman K. Modern Epidemiology. Boston/Toronto: Little, Brown \& Company. 1986. pp. 430.

9.- Giesecke J. Modern Infections Disease Epidemiology. Edward Arnold. London: 1994. p. 97-108.

10.- Martin W. Veterinary epidemiology: principles and methods. Ames: Iowa State Univ. Press-Ames; 1987. p. 435 .

11.- Inegi. Anuario estadístico del estado de Yucatán: Climatología del estado de Yucatán. Mérida: INEGIGob. del Edo. de Yucatán; 1996. p. 11-21.

12.- Panacea. ODA Proyect IBM \& compatible version Copy Right, 1989. Reading: Panlivestock Service Limited; 1989. p. 215.

13.- Aguilar CAJ. Principales causas de mortalidad en lechones en predestete en la granja " 00 " de Quintana Roo. Tesis de Licenciatura en Medicina Veterinaria y Zootecnia. FMVZ-UADY. Mérida Yucatán, México. 1992. p. 42.

14.- Mack S. Evaluation of the productivities of west African dwarf sheep and goats. Humid Zone Programme,
ILCA. Doc No. 7. Ibadan; 1983. p. 37-55.

15.- Opasina, B. Disease constraints on village goat production in southwest Nigeria. International Livestock Centre for Africa (ILCA). Ibdan, Nigeria; 1985. p. 1139.

16.- Smith O, Van-Houter M. Health aspects of intensive management of west African Dwarf goats in the humid forest zone. Procc. National Conference Disease of ruminants. Vom, Nigeria; 1994. p. 7-22.

17.- O'Brien JP, Sherman DM. Serum immunoglobulin concentrations of newborn goat kids and subsequent kid survival through weaning. Small Rumin Res 1993; 11:71-7.

18.- Lodh C, Chakraborti A, Mukhopadhyay V. Factors affecting kid mortality in West Bengal. Indian Vet $\mathrm{J}$ 1993; 70:48-50.

19.- Lemos MJ, Almeida JE, De-Almeida JE A. A survey of goat breeding in Sao Paulo State. Zootecnia Sao Paulo 1993; 31:29-46.

20.- Ba SB, Udo HMJ, Zwart D. Impact of veterinary treatments on goat mortality and offtake in the semiarid area of Mali. Small Rumin Res 1996; 19:1-8.

21.- Devendra C, Burns M. Goat production in the tropics. CAB. Farmham Royal. 1983. p. 88-89.

22.- Morand-Fehr P. Growth. In: Gall C. Goat production. N. York: Acadec Press. Inc; 1991. p. 25383.

23.- Slee, J. A review of genetic aspects of survival and resistance to cold in newborn lambs. Livestock Prod Sci 1981; 8:419-29.

24.- Moran-Ferhr P. Growth. In Gall C. Goat Production. New York: Academic Press; 1981. p. 23-31.

25.- Sykes A. Nutrition an parasites. Recent advances on the nutrition of herbivores. Canterbury: Lincoln University Canterbury; 1991. p. 26-38.

26.- Torres AJF, Aguilar CAJ, Rodríguez RI. Efecto de la Ivermectina sobre la ganancia de peso de cabritos criollos en clima tropical. Memorias de la XI Reunión Nacional sobre Caprinocultura. UACH-AMPCA. Edo. Méx., México; 1996. p.159-163. 
Mortalidad de cabras en Yucatán, México.

27.- Campos RR, Herrera RD, Quiroz RH. Diagnóstico in vitro de Haemonchus contortus resistente al albendazol, febendasol, oxfendazol y febantel en tres rebaños ovinos Tabasco o Pelibuey. Vet Méx 1992; 23:516.

28.- Coles GC, Roush RT. Slowing the spread of anthelmintic resistance nematodes of sheep and goats in United Kingdow. Vet Rec 1992; 130:505-2.

29.- Pomroy WE, Charleston WAG. Failure of young goats to acquire resistance to Haemonchus contortus. NZvet J 1989; 37:23-6.

30.- Al-Quaisy, HHK, Al-Subaidy AJ, Altaif K.I, Makkawi TA. The pathogenicity of Haemonchosis in Sheep and goats in Iraq: 1. Clinical, parasitology and haematological findings. Vet Parasitol 1987; 24:221-8. 